\title{
Quadratic Optical Frequency Combs: Towards a New Platform for Multi-Octave Microcombs
}

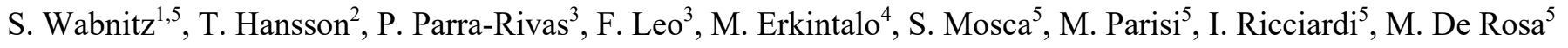 \\ ${ }^{1}$ DIET, Sapienza University of Rome, Rome, Italy \\ ${ }^{2}$ Department of Physics, Chemistry and Biology, Linköping University, Linköping, Sweden \\ ${ }^{3}$ OPERA-Photonics, ULB, Brussels, Belgium \\ ${ }^{4}$ Department of Physics, University of Auckland, Auckland, New Zealand \\ ${ }^{5}$ CNR-INO, Istituto Nazionale di Ottica, Pozzuoli, Italy
}

\begin{abstract}
Optical frequency comb sources based on threewave-mixing in quadratic nonlinear materials allow for reduced pump power threshold and extended spectral coverage. We review recent progress on quadratic optical frequency combs based on second-harmonic generation and optical parametric oscillation.
\end{abstract}

Keywords- Optical frequency combs; second-harmonic generation; optical parametric oscillation; optical solitons

\section{INTRODUCTION}

The development of optical frequency combs (OFCs) has a strong impact on metrology and spectroscopy. Recent advances in optical micro-cavity technologies have enabled many demonstrations of chip-scale OFC sources, primarily based on Kerr nonlinearities [1]. Open challenges remain, in terms of the threshold pump power, conversion efficiency from the pump to the comb, comb stabilization, scarcity of pump sources in certain spectral regions, etc.

A solution to these issues could be provided by OFC sources based on quadratic, as opposed to cubic, nonlinear resonators. Here, we review our theoretical and experimental progress in the field of quadratic OFCs.

\section{SHG COMBS}

In 2015, Ricciardi et al. demonstrated the direct generation of OFCs in a cavity-enhanced second-harmonic generation (SHG) system [2]. Two OFCs were simultaneously generated around the fundamental (FF) pump frequency at $1064 \mathrm{~nm}$ and its second harmonic ( $\mathrm{SH})$. A modal expansion comb model was later developed [3]. In 2016, Leo et al. derived a single timedomain, mean-field equation that models OFC generation in singly resonant, cavity SHG systems [4]. SHG OFCs originate from a wallk-off induced modulation instability (MI), and have a rich temporal and spectral dynamics [5]. OFC generation in doubly-resonant intracavity SHG was also analyzed by Leo et al. [6]. OFC dynamics can be well modeled by a pair of coupled mean-field equations; under certain conditions, these can be reduced to a single mean-field equation, similar to the singly resonant-cavity SHG case. A study by Hansson et al. predicted, in the absence of temporal walk-off between the FF and the SH, the generation of both bright and dark time-localized cavity soliton combs [7]. These soliton solutions correspond to stable, broadband and coherent OFCs.

This work was funded by: Ministero dell'Istruzione, Università e Ricerca (2015KEZNYM-NEMO); H2020 European Research Council (ERC) (757800).

\section{OPO COMBS}

In 2018, Mosca et al. reported on the formation of quadratic combs in an optical parametric oscillator (OPO) [8]. OFCs were generated around half of the pump frequency in a singlyresonant, CW-driven OPO. The wave dynamics could be modeled by means of a single time-domain mean-field equation: the dynamical regimes leading to OFC formation were identified. The formation of localized temporal dissipative structures, and their corresponding OFCs, in doubly-resonant dispersive OPOs, was also recently investigated by Parra Rivas et al. [9].

\section{REFERENCES}

[1] A. Pasquazi, M. Peccianti, L. Razzari, D. J. Moss, S. Coen, M. Erkintalo, Y. K. Chembo, T. Hansson, S. Wabnitz, P. Del'Haye, X. Xue, A. M. Weiner, and R. Morandotti, "Micro-combs: A novel generation of optical sources," Physics Reports, vol. 729, pp. 1-81, 2018.

[2] I. Ricciardi, S. Mosca, M. Parisi, P. Maddaloni, L. Santamaria, P. De Natale, and M. De Rosa, "Frequency comb generation in quadratic nonlinear media," Phys. Rev. A, vol. 91, p. 063839, June 2015.

[3] S. Mosca, I. Ricciardi, M. Parisi, P. Maddaloni, L. Santamaria, P. D. Natale, and M. D. Rosa, "Direct generation of optical frequency combs in $\chi^{2}$ nonlinear cavities," Nanophotonics., vol. 5, no. 2, pp. 361-331, June 2016 .

[4] F. Leo, T. Hansson, I. Ricciardi, M. De Rosa, S. Coen, S. Wabnitz, and M. Erkintalo, "Walk-off-induced modulation instability, temporal pattern formation, and frequency comb generation in cavity-enhanced secondharmonic generation," Phys. Rev. Lett., vol. 116, p. 033901, January 2016.

[5] T. Hansson, F. Leo, M. Erkintalo, S. Coen, I. Ricciardi, M. De Rosa, and S. Wabnitz, "Singly resonant second-harmonic-generation frequency combs,” Phys. Rev. A, vol. 95, p. 013805, January 2017.

[6] F. Leo, T. Hansson, I. Ricciardi, M. De Rosa, S. Coen, S. Wabnitz, and M. Erkintalo, "Frequency-comb formation in doubly resonant secondharmonic generation,” Phys. Rev. A, vol. 93, p. 043831, April 2016.

[7] T. Hansson, P. Parra-Rivas, M. Bernard, F. Leo, L. Gelens, and S. Wabnitz, "Quadratic soliton combs in doubly resonant secondharmonic generation,” Opt. Lett., vol. 43, no. 24, pp. 6033-6036, December 2018.

[8] S. Mosca, M. Parisi, I. Ricciardi, F. Leo, T. Hansson, M. Erkintalo, P. Maddaloni, P. De Natale, S. Wabnitz, and M. De Rosa, "Modulation instability induced frequency comb generation in a continuously pumped optical parametric oscillator," Phys. Rev. Lett., vol. 121, p. 093903, August 2018.

[9] P. Parra-Rivas, L. Gelens, T. Hansson, S. Wabnitz, and F. Leo, "Frequency comb generation through the locking of domain walls in doubly resonant dispersive optical parametric oscillators," Opt. Lett., vol. 44, no. 8, pp. 2004-2007, April 2019. 